(c) 2016 IEEE. Personal use of this material is permitted. Permission from IEEE must be obtained for all other uses, in any current or future media, including reprinting/republishing this material for advertising or promotional purposes, creating new collective works, for resale or redistribution to servers or lists, or reuse of any copyrighted component of this work in other works.

DOI: 10.1109/EuMC.2016.7824267 


\title{
Design and Experimental Characterization of a Surface with Low Radar Cross-Section at Millimeter-Wave Frequencies
}

\author{
Claudia Vasanelli, Frank Boegelsack, and Christian Waldschmidt, \\ Ulm University, Institute of Microwave Engineering, 89081 Ulm, Germany \\ E-mail: forename.surname@uni-ulm.de
}

\begin{abstract}
The investigation of low radar cross-section (RCS) structures is of great practical interest for the car integration of radar systems. This paper presents for the first time the study of a low-RCS surface at millimeter wave frequencies. The surface employs a chessboard-like geometry that uses a periodic displacement of artificial magnetic conductors (AMCs) and perfect electric conductors (PECs). The structure is able to redirect the incident wave in non-specular directions, and it is thus possible to achieve a significant reduction of the RCS in the boresight direction. The measurement results confirm the predictions of the full-wave simulations, and they prove that the proposed surface can effectively achieve more than $17 \mathrm{~dB}$ of RCS reduction in the case of normal incidence at $76.8 \mathrm{GHz}$.
\end{abstract}

\section{INTRODUCTION}

Millimeter-wave technology is widely employed in a number of applications for both communication and radar purposes. In particular, over the last few years an increased number of radar sensors has been designed to work in the $76-81 \mathrm{GHz}$ frequency range that has been assigned in Europe to automotive radar for driving assistance systems.

Usually in such applications the sensor is mounted behind the bumper of the car. The bumper is a complex multilayer structure, whose materials have different values of permittivities in the range from 2 to 8 [1]. Moreover, the external coating often contains metal particles and this makes challenging the integration of the sensor. Part of the energy transmitted by the sensor will be indeed reflected back by the bumper. The reflected wave will impinge then on the metal housing and on the printed circuit board (PCB) of the radar sensor. The receiving antennas will intercept this reflected wave, which will be also again reflected towards the bumper. These multiple reflections potentially lead to a wrong estimation of the position of the target or angular ambiguities [2], causing for example a wrong braking intervention. Therefore, the investigation of proper techniques to mitigate the multiple reflections between the bumper and the sensor is of great practical interest.

A possible solution to mitigate the reflections can be the use of different coatings or the use of proper matching layers, as presented in [2]. Unfortunately, this solution is not feasible, since the bumper must follow specific safety requirements and it is a key element of the aesthetic guidelines of car manufacturers. In the end, minimizing the reflections between the radar sensor and the bumper means essentially to design a sensor with a low RCS. Indeed, the RCS describes the amount of backscattered power when a target is illuminated by an incident plane wave [3]. Techniques for RCS reduction have been already extensively investigated especially for military applications. The most common approaches to reduce the RCS of a structure are the use of radar absorbing material or the proper design of the shape of the structure to redirect the scattered fields [4].

It is important to notice that the reduction of the RCS of the sensor must be fully compatible with standard PCB fabrication process to avoid any increase in the complexity of the design or in the production costs.

Interesting low-RCS surfaces have been recently proposed in [4] and [5]. They are based on the use of a periodic displacement of AMCs and metallic patches in a chessboard-like geometry. The theoretical and experimental results prove that this kind of surface can effectively achieve a significant RCS reduction compared to an equal-sized PEC. These structures are promising candidates for the integration into an automotive radar sensor.

To the best of the authors' knowledge, chessboard-like lowRCS surfaces have never been designed and experimentally characterized at millimeter-wave frequencies. In this work, for the first time a low-RCS structure is proposed at $77 \mathrm{GHz}$. The use of higher frequencies poses specific challenges, mainly related to the increased etching tolerances, to losses in the PCB technology, and to the measurement setup.

This paper is organized as follows. The principle of operation of the proposed low-RCS surface is described in Section II. Section III discusses first the measurement setup and then presents the experimental results of a fabricated prototype. Finally, Section IV summarizes the main results of this work.

\section{Principle of Operation AND Design of the SURFACE}

As can be seen in Fig. 1, a low-RCS chessboard surface uses a combination of AMC and PEC cells to achieve a reduction of the backscattered wave in the boresight direction. As extensively described in [6], an AMC at the resonance frequency mimics the behavior of a magnetic conductor and shows a reflection phase of $0^{\circ}$, i.e. the reflection coefficient is +1 . Since the reflection phase of a PEC is $180^{\circ}$, i.e. the reflection coefficient is -1 , the fields reflected by AMC and 


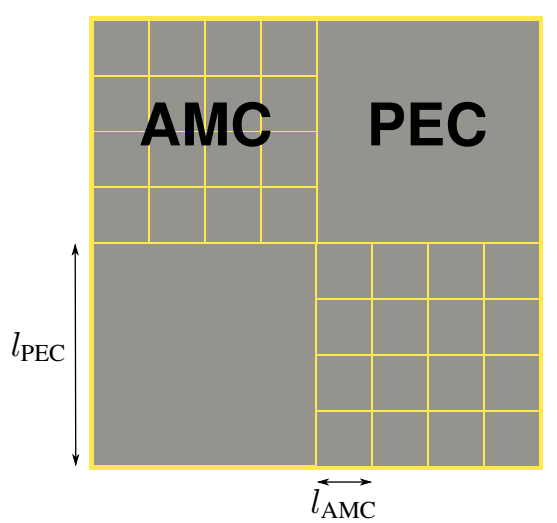

Fig. 1. Unit cell of the chessboard surface.

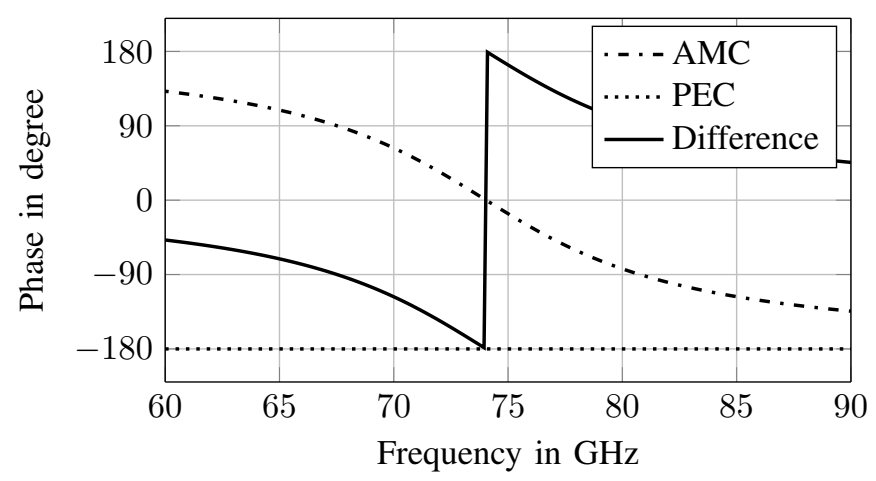

Fig. 2. Reflection phase of the AMC and PEC cells and phase difference between them.

PEC surfaces are $180^{\circ}$ out of phase and therefore, destructive interference takes place. The incident field will not be scattered in the boresight direction, instead it will be redirected in different directions that can be controlled by changing the dimension of the unit cell [4], [7].

In this work, the first investigations are conducted by using a square metal patch as AMC with a side length $l_{\mathrm{AMC}}$ of $0.88 \mathrm{~mm}$ as depicted in Fig. 1. The distance between two adjacent patches is $0.14 \mathrm{~mm}$. The PEC has a side length $l_{\mathrm{PEC}}$ of $3.94 \mathrm{~mm}$, thus the total dimensions of the unit cell presented in Fig. 1 are $8.16 \mathrm{~mm} \times 8.16 \mathrm{~mm}$. The structure is designed on RO3003 substrate from Rogers Corporation [8] with $127 \mu \mathrm{m}$ thickness and with the relative permittivity $\varepsilon_{r}=3$.

Before performing a full-wave simulation of the chessboard structure, the approximated model presented in [4] for the evaluation of the RCS reduction has been used. Since the surface is composed of two subsections, namely the AMC and the PEC, with equal dimensions, the RCS reduction can be calculated by taking the average of the reflection coefficient of the two subsections. Thus, the approximated reduction of the RCS can be estimated with

$$
r=10 \log \left|\frac{A_{1} \mathrm{e}^{\mathrm{j} P_{1}}+A_{2} \mathrm{e}^{\mathrm{j} P_{2}}}{2}\right|^{2} \mathrm{~dB},
$$

where $A_{1}$ and $A_{2}$ are the amplitudes of the reflection coefficients and $P_{1}$ and $P_{2}$ the phases. In Fig. 2 the reflection phase

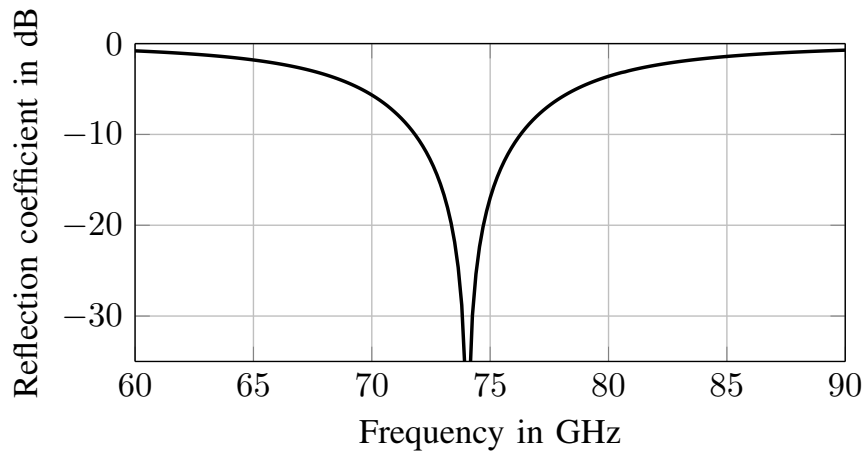

Fig. 3. Approximated RCS reduction according to formula (1).

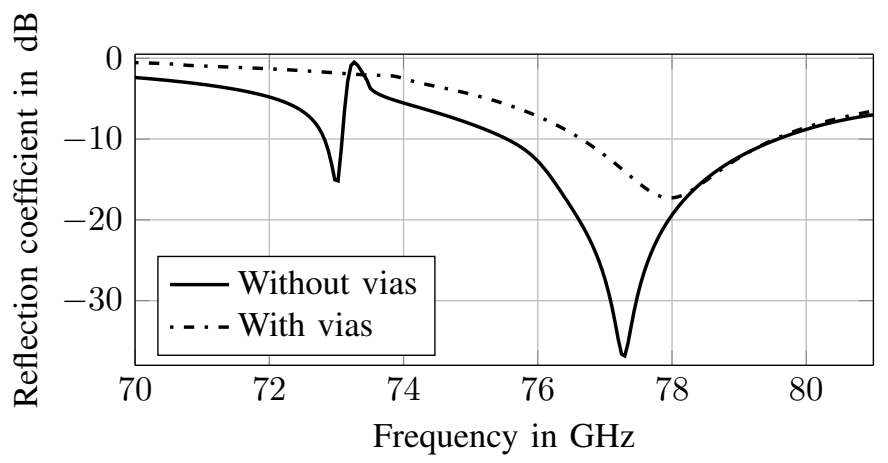

Fig. 4. Comparison of the low-RCS surfaces that employ square metal patches and grounded square metal patches as AMC.

of the AMC and PEC are shown. When $180^{\circ}$ phase difference is achieved and assuming that the amplitudes $A_{1}$ and $A_{2}$ are equal, a minimum in the amplitude of the reflection coefficient can be observed, as reported in Fig. 3.

Two low-RCS surfaces have been designed and investigated. The proposed structures have been simulated by means of a 3D full-wave simulation software. To simulate an incident plane wave impinging on the structure, periodic boundary conditions have been applied. In this way it is possible to simulate an infinitely extent of the structure and the edge effects are neglected. To simplify the numerical model, the metal layers are considered ideal.

The first model has been previously described and it employs a square patch as AMC. The second one uses a square patch (length of the side $0.96 \mathrm{~mm}$ ) connected to the ground by a vertical via (with $0.3 \mathrm{~mm}$ diameter) as AMC [6]. The comparison of the two structures is presented in Fig. 4. The plot shows that both structures can reduce the RCS in the frequency range of interest, but the use of AMCs without vias allows to widen the bandwidth. As can been seen, the bandwidth where the amplitude of the reflection coefficient is below $-10 \mathrm{~dB}$ is approximately $4 \mathrm{GHz}$. Due to the larger bandwidth, only the surface without grounding vias will be further investigated and characterized.

Figure 5 shows the simulated 3D bistatic RCS pattern of the proposed structure in case of normal incidence. The plot 


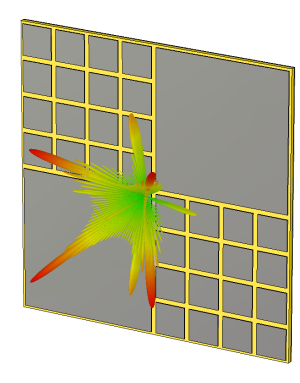

in $\mathrm{dB}$

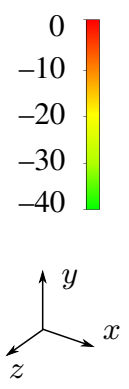

Fig. 5. Simulated 3D bistatic RCS at $77 \mathrm{GHz}$.

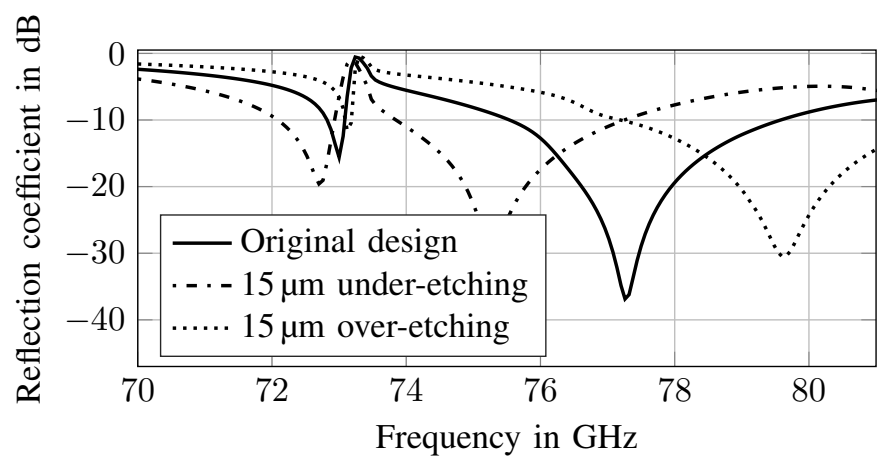

Fig. 6. Effect of a possible PCB fabrication error on the performance of the low-RCS surface.

clearly explains that the impinging wave is backscattered in four main different beams and a minimum in the value of the $\mathrm{RCS}$ can be seen in the boresight direction.

Fabrication inaccuracies might become critical at millimeter-wave frequencies. Under- and over-etching of the PCB leads to both a shift in the minimum of the reflection coefficient and to different directions of the backscattered beams. Moreover, the proposed surface is composed of many long thin slots that divide the AMC and PEC cells; it is a well-known problem that such a structure can hardly be realized accurately with standard photo-etching process at high frequencies. So, it is interesting to model also in the full-wave simulation an etching error to understand how sensitive the chessboard-like structure is. As an example, only the worst case scenario has been considered, i.e. $15 \mu \mathrm{m}$ etching tolerances. As can be seen in Fig. 6, the fabrication inaccuracy causes a frequency shift of less than $3 \%$ with respect to the results obtained with the ideal design. Additionally, even in the worst cases it is possible to achieve at least $5 \mathrm{~dB}$ of $\mathrm{RCS}$ reduction in the frequency range of interest.

\section{EXPERIMENTAL RESUlTS}

The fabricated prototype can be seen in Fig. 7. It is composed of $10 \times 10$ unit cells. To increase the mechanical stability of the structure, an FR-4 support has been placed on the back side. The visual inspection showed no more than $15 \mu \mathrm{m}$ etching tolerances.

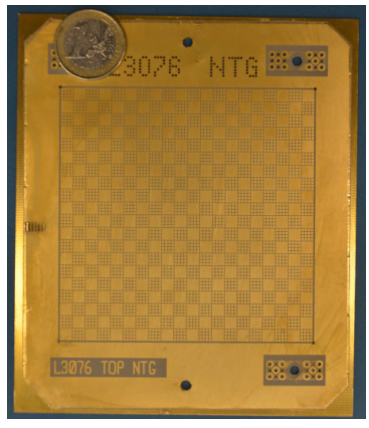

(a)

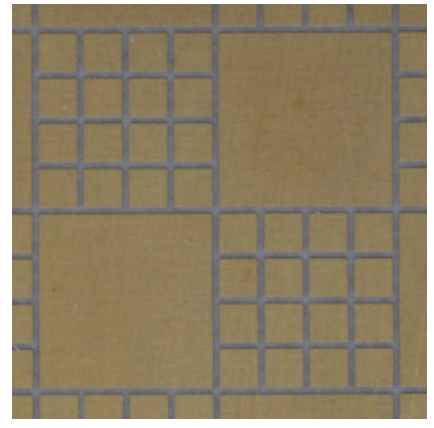

(b)
Fig. 7. (a) Fabricated prototype and (b) enlarged view of one unit cell.

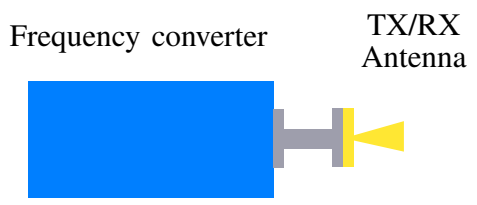

Waveguide
SUT/PEC

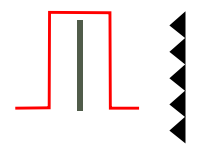

Absorber
Fig. 8. Schematic of the measurement setup. The red window describes the bandpass filter applied during the time gating procedure.

\section{A. Measurement Setup}

The measurements have been conducted in an anechoic chamber using a monostatic setup. The surface under test (SUT) has been place on a turntable. At a distance of approximately $3.3 \mathrm{~m}$ a $25 \mathrm{dBi}$ standard-gain horn antenna connected to a frequency converter module has been placed on a tripod at the same height as the SUT. The frequency converter module allows to extend the frequency range of the vector network analyzer (VNA) up to the E-band $(60-90 \mathrm{GHz})$. The edges of the SUT and the turntable have been covered with absorbing material to suppress any spurious reflection.

A schematic of the measurement setup is depicted in Fig. 8. Two successive measurements have been performed: in the first one the SUT is placed on the turntable, in the second one an equal-sized copper plate has been used. This configuration permits an easy and fair evaluation of the RCS reduction properties of the SUT in comparison to a PEC plate.

As can be easily understood, due to the small amplitude of the collected signal it is important to maximize the dynamic range of the VNA for the characterization of the SUT. Consequently, the IF bandwidth has been reduced to $100 \mathrm{~Hz}$, and the output power has been set to $4 \mathrm{dBm}$.

To collect only the signal back-scattered by the SUT the time gating has been applied. The frequency span has been set to $30 \mathrm{GHz}$ to maximize the response resolution by using the time gating; with $6 \mathrm{~cm}$ bandpass window width the response resolution is approximately $9.7 \mathrm{~mm}$ for reflection measurements [9].

Finally, it is worth to underline the importance of a correct alignment between the horn antenna and the SUT. In this mea- 


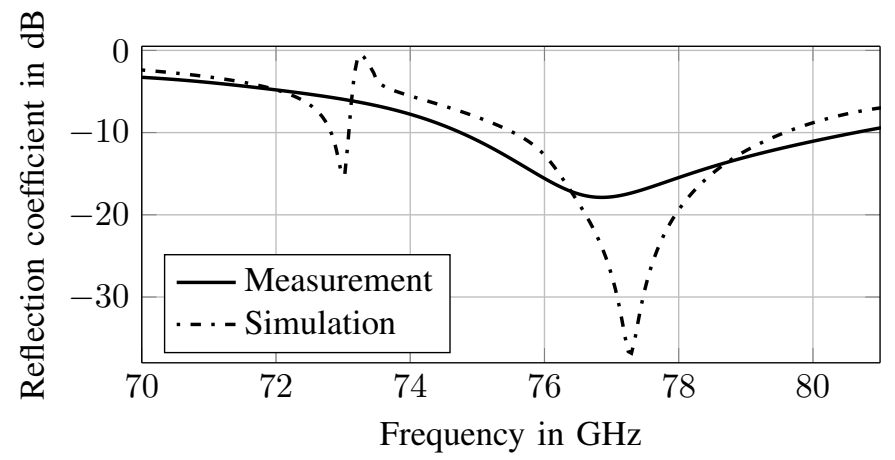

Fig. 9. Comparison of the measured and simulated reflection coefficient for the normal incidence.

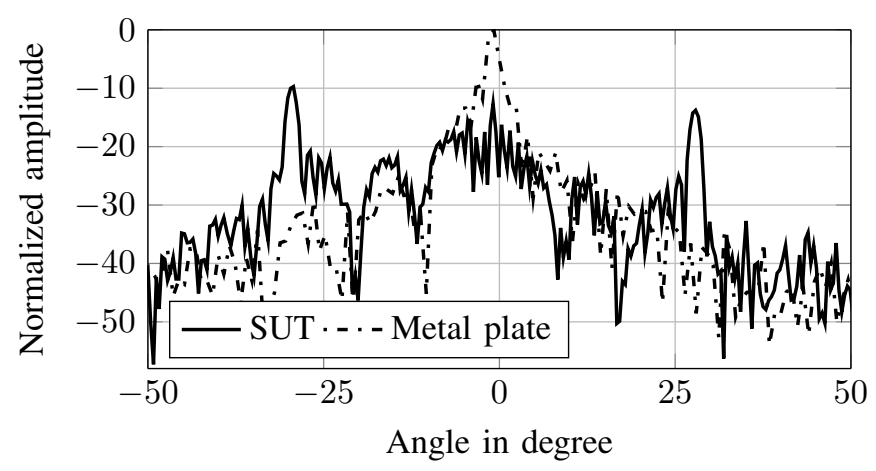

Fig. 10. Measured monostatic normalized RCS pattern at $76.8 \mathrm{GHz}$.

surement setup only a manual alignment is performed with the help of a laser pointer, similarly to what is described in [10]. A misalignment can cause a different appearance direction of the grating lobes from the theoretical values predicted by the array theory or by the full-wave simulations [7].

\section{B. Evaluation of the RCS Reduction}

Figure 9 depicts the comparison between the measured and simulated reflection coefficient versus frequency of the chessboard-like structure in the case of normal incidence. It can be noticed that a good agreement is obtained. Due to the fabrication inaccuracies, the measured minimum of the reflection coefficient is shifted by less than $1 \mathrm{GHz}$ towards lower frequencies with respect to the simulation results. The measured bandwidth is approximately $6 \mathrm{GHz}$ centered around $76.8 \mathrm{GHz}$. The measurement proves that the proposed structure is able to achieve $17 \mathrm{~dB}$ of $\mathrm{RCS}$ reduction at $76.8 \mathrm{GHz}$ in the boresight direction.

As explained in the previous section, during the measurements the SUT has been placed on a turntable, therefore it is also possible to evaluate the RCS for the case of oblique incidence by turning the structure on the azimuth plane. The measurement results are reported in Fig. 10 for $76.8 \mathrm{GHz}$. This plot confirms again the effective reduction of the RCS at $0^{\circ}$ compared to an equal-sized metal plate. It can also be observed that the incident wave is now backscattered in

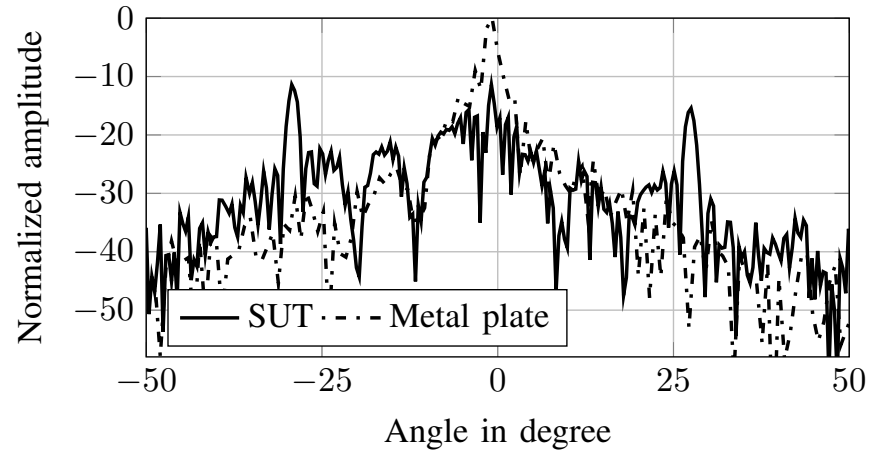

Fig. 11. Measured monostatic normalized RCS pattern at $78 \mathrm{GHz}$.

different directions, in particular two beams can be clearly seen at $-29^{\circ}$ and at $+27^{\circ}$.

As an example, Fig. 11 shows the measured monostatic RCS pattern at $78 \mathrm{GHz}$. Also in this case, the amplitude of the RCS in the boresight direction is significantly reduced compared to the metal plate.

\section{CONCLUSION}

This paper presents the investigation and the characterization of a low-RCS surface that could be potentially integrated in an automotive radar sensor to mitigate the reflections caused by the presence of the bumper.

The numerical and experimental results show that the proposed structure can successfully reduce the RCS in case of normal incidence by more than $17 \mathrm{~dB}$ compared to an equalsized PEC at $76.8 \mathrm{GHz}$. Moreover, the surface is able to reduce the RCS in the boresight direction by more than $10 \mathrm{~dB}$ in a $6 \mathrm{GHz}$ bandwidth.

\section{REFERENCES}

[1] R. Schnabel, D. Mittelstrab, T. Binzer, C. Waldschmidt, and R. Weigel, Reflection, Refraction, and Self-Jamming, in IEEE Microwave Magazine, vol. 13, no. 3, pp. 107-117, 2012.

[2] F. Fitzek, R. H. Rashofer, and E. M. Biebl, Broadband matching of high-permittivity coatings with frequency selective surfaces, in German Microwave Conf. (GeMIC), pp. 1-4, 14-16 March 2011.

[3] E. F. Knott, J. F. Shaeffer, and M. T. Tuley, Radar Cross Section, 2nd. Ed., Scitech Publishing, Inc., Raleigh, NC, 2004.

[4] W. Chen, C. A. Balanis, and C. R. Birtcher, Checkerboard EBG Surfaces for Wideband Radar Cross Section Reduction, in IEEE Trans. Antennas Propag., vol. 63, no. 6, pp. 2636-2645, June 2015.

[5] M. Paquay, J.-C. Iriarte, I. Ederra, R. Gonzalo, and P. de Maagt, Thin AMC Structure for Radar Cross-Section Reduction, in IEEE Trans. Antennas Prop., vol. 55, no. 12, pp. 3630-3638, Dec. 2007.

[6] D. Sievenpiper, L. Zhang; R. F. J. Broas, N. G. Alexopolous, and E. Yablonovitch, High-impedance electromagnetic surfaces with a forbidden frequency band, IEEE Trans. Microw. Theory Tech., vol. 47, no. 11, pp. 2059-2074, Nov. 1999.

[7] J.C. Iriarte Galarregui, A. Tellechea Pereda, J. L. Martinez de Falcon, I. Ederra, R. Gonzalo, and P. de Maagt, Broadband Radar Cross-Section Reduction Using AMC Technology, IEEE Trans. Antennas Prop., vol. 61, no. 12, pp. 6136-6143, Dec. 2013.

[8] Rogers Corporation, CT 06263, USA.

[9] Agilent Technologies, Agilent Time Domain Analysis Using a Network Analyzer, Application Note 1287-12, pp. 29-33.

[10] Y. Alvarez, M. E. de Cos, and F. Las-Heras, RCS Measurement Setup for Periodic-Structure Prototype Characterization, in IEEE Antennas Prop. Magazine, vol. 52, no. 3, pp. 100-106, June 2010. 